\title{
Strain-rate formulation of ice fabric evolution
}

\author{
LESLIE W. MORLAND, RYsZARD STAROSZCZYK \\ School of Mathematics, University of East Anglia, Norwich NR4 7T J, England \\ E-mail: l.morland@uea.ac.uk
}

\begin{abstract}
Reorientation of individual crystal-glide planes as isotropic surface ice is deformed during its passage to depth in an ice sheet, lattice rotation, creates a fabric and associated anisotropy. A simple macroscopic description is that these material glide planes are rotated towards planes normal to an axis of compression, and away from planes normal to an axis of extension, inducing an instantaneous orthotropic viscous response with reflexional symmetries in the planes orthogonal to the current principal stretch axes. An orthotropic viscous law is presented for the strain rate expressed in terms of the deviatoric stress, the deformation, and three structure tensors based on the principal stretch axes. This anisotropic relation is expressed in terms of a single fabric response function in addition to the isotropic ice viscosity. The predicted responses in uniaxial compression and simple shear are determined. While the uniaxial response yields an explicit relation between the axial strain rate and stress, it is found that the shear response is governed by three, complicated, coupled relations between the shear strain rate and three deviatoricstress components. The new result derived here is the solution of this system: an explicit relation between the shear strain rate and shear stress. Correlation of these relations with idealized uniaxial and shear responses is then used to determine the required fabric function in the model law.
\end{abstract}

\section{INTRODUGTION}

Ice-core samples taken from depth in an ice sheet reveal strong fabrics, shown by significant alignment of initially randomly distributed $c$ axes of individual crystals, and consequent substantial differences in shear viscosities in different planes. Macroscopic laws have been constructed from the properties of individual crystals and assumptions on how they interact (e.g. by Van der Veen and Whillans, 1994; Azuma and Goto-Azuma, 1996). Visco-plastic self-consistent theory has been applied (e.g. by Castelnau and others, 1996; Meyssonnier and Philip, 1996) to derive average properties. Meyssonnier and Philip (1996) also introduced an orientation distribution function to measure weightings of a continuous spectrum of $c$-axis orientations, and this approach has been used to construct direct macroscopic laws (e.g. by Svendsen and Hutter, 1996; Gödert and Hutter, 1998; Gagliardini and Meyssonnier, 1999).

An alternative macroscopic viscous law for the shear stress motivated by a simple picture of lattice rotation in which individual crystal-glide planes, material planes, are rotated towards planes normal to principal axes of compression, and away from planes normal to principal axes of extension, has been developed by Morland and Staroszczyk (1998, 2003) and Staroszczyk and Morland (2000, 2001). Instantaneous directional viscosities were introduced to define the induced anisotropy in any given fabric state, and a set of equalities and inequalities which must be satisfied by the instantaneous directional viscosities was derived from the above rotation concepts, designated by one author (L.W.M.) as the "Staroszczyk inequalities". It was inferred that the instantaneous viscous response is orthotropic with respect to the current principal stretch planes, so that the base planes of the orthotropy are evolving, and that the directional strengths of the response depend on the current deformation. The orthotropic viscous law is then a frame-indifferent relation between stress, strain rate, deformation and the three structure tensors defined by the outer products of the three orthogonal vectors along the principal stretch axes.

Morland and Staroszczyk (2003) analyzed the simplest forms of both stress and strain-rate formulations, relations for the deviatoric stress and for the strain rate, respectively, consistent with the Staroszczyk inequalities, which each involve two sets of tensor generators but only one independent fabric response coefficient depending on one invariant argument. The stress formulation yielded explicit relations between the primary stress and strain-rate components in both uniaxial compression and simple shear responses, and correlations between the predicted and assumed idealized responses were made to determine the corresponding fabric response function. For the strain-rate formulation, an explicit relation between the axial strain rate and stress was again obtained in uniaxial compression; in simple shear, however, the shear strain rate is governed by three coupled relations in terms of the shear stress and two axial deviatoric stresses, and involves the fabric response function in a complicated, non-linear manner. Correlations with the idealized responses did not appear feasible. We now re-examine the Morland and Staroszczyk (2003) strain-rate formulation, and determine a crucial new result: the solution of this coupled system to derive an explicit relation for the shear strain rate in terms of the shear stress. We then determine the fabric response function for the strain-rate formulation by correlation with the same idealized uniaxial and shear 
responses adopted by Morland and Staroszczyk (2003) for the stress formulation. Only relations necessary to describe the model and responses, and the new solution, are repeated.

\section{ORTHOTROPIC VISGOUS MODEL}

We adopt the orthotropic viscous law for the strain rate $\mathbf{D}$ presented by Morland and Staroszczyk (2003),

$$
\begin{aligned}
2 \mu(J) \tilde{\mathbf{D}} & =\left\{\hat{\boldsymbol{\sigma}}+\sum_{r=1}^{3} \tilde{f}\left(\xi_{r}\right)\left[\mathbf{M}^{(r)} \hat{\boldsymbol{\sigma}}+\hat{\boldsymbol{\sigma}} \mathbf{M}^{(r)}\right.\right. \\
& \left.-\frac{2}{3} \operatorname{tr}\left(\mathbf{M}^{(r)} \hat{\boldsymbol{\sigma}}\right) \mathbf{I}\right] \\
& \left.+\tilde{g}(\eta)\left[\hat{\boldsymbol{\sigma}} \mathbf{B}+\mathbf{B} \hat{\boldsymbol{\sigma}}-\frac{2}{3} \operatorname{tr}(\hat{\boldsymbol{\sigma}} \mathbf{B}) \mathbf{I}\right]\right\}, \mathbf{D}=a(T) \tilde{\mathbf{D}},
\end{aligned}
$$

where $\mathbf{I}$ is the unit tensor, $T$ is temperature, $\tilde{\mathbf{D}}$ is an effective strain rate incorporating a temperature-dependent rate factor $a(T)$, and $\mathbf{B}$ is the left Cauchy-Green strain tensor. $\hat{\boldsymbol{\sigma}}$ is the deviatoric-stress tensor with a dimensionless invariant $J$ relative to a typical ice-sheet flow shear stress magnitude $\sigma_{0}$ :

$$
J=\frac{1}{2} \operatorname{tr}\left(\frac{\hat{\boldsymbol{\sigma}}}{\sigma_{0}}\right)^{2}, \quad \sigma_{0}=10^{5} \mathrm{~N} \mathrm{~m}^{-2} .
$$

$\mu(J)$ is the isotropic viscosity function. $\tilde{f}\left(\xi_{r}\right), \tilde{g}(\eta)$ are fabric response coefficients, functions of single deformation invariant arguments $\xi_{r}$ and $\eta$, respectively, given by

$\xi_{r}=b_{r}-1 / b_{r}(r=1,2,3), \quad \eta=\sqrt{(K-1)^{2}-4}$,

$K=\operatorname{tr} \mathbf{B}=b_{1}+b_{2}+b_{3}, \quad b_{1} \geq b_{2} \geq b_{3}$,

where $b_{r}(r=1,2,3)$ are the ordered principal values (principal stretches squared) of B. $\tilde{f}\left(\xi_{r}\right)$ and $\tilde{g}(\eta)$ are supposed differentiable functions of their arguments. The three structure tensors $\mathbf{M}^{(r)}$ are defined by the outer products of the unit vectors $\mathbf{e}^{(r)}(r=1,2,3)$ along the current principal stretch axes:

$$
\mathbf{M}^{(r)}=\mathbf{e}^{(r)} \otimes \mathbf{e}^{(r)}, \quad(r=1,2,3) .
$$

Isotropy in the undeformed state $\mathbf{B}=\mathbf{I}$ gives the normalization condition

$$
\tilde{f}(0)+\tilde{g}(0)=0 .
$$

The non-trivial equality of the general Staroszczyk inequalities requires

$$
\tilde{G}(\eta)=\eta \tilde{g}(\eta)=-\tilde{f}(\eta)+\tilde{f}(-\eta)=-2 \tilde{f}^{\circ}(\eta),
$$

for $\eta \geq 0$, where $\tilde{f}(\xi)$ has the decomposition into even, $\tilde{f}^{\mathrm{e}}$, and odd, $\tilde{f}^{\circ}$, parts defined by

$$
2 \tilde{f}^{\mathrm{e}}(\xi)=\tilde{f}(\xi)+\tilde{f}(-\xi), 2 \tilde{f}^{\mathrm{o}}(\xi)=\tilde{f}(\xi)-\tilde{f}(-\xi),
$$

and the alternative fabric response function $\tilde{G}(\eta)$, defined by Equation (2.6), is finite and non-zero as $\eta \rightarrow \infty$. The limit of Equation (2.6) as $\eta \rightarrow 0$, noting that $\tilde{f}^{\circ}(0) \sim \eta \tilde{f}^{\prime}(0)$, together with Equation (2.5), shows that

$$
\tilde{G}(0)=0, \quad \tilde{g}(0)=-\tilde{f}(0)=-2 \tilde{f}^{\prime}(0),
$$

which is a restriction on $\tilde{f}(\xi)$ at $\xi=0$. Thus $\tilde{g}(\eta)$ and $\tilde{G}(\eta)$ are expressed explicitly in terms of $\tilde{f}^{\circ}(\eta)$, and the constitutive law given by Equation (2.1) can be expressed in terms of a single independent fabric response function $\tilde{f}(\xi)$. Define, for $(i, j=1,2,3 ; i \neq j)$,

$$
\tilde{h}_{i j}=\tilde{f}\left(\xi_{i}\right)-\tilde{f}\left(\xi_{j}\right)-2 \eta^{-1} \tilde{f}^{o}(\eta),
$$

then the remaining non-trivial Staroszczyk inequalities require

$$
\tilde{h}_{21}<0, \quad \tilde{h}_{23}<0, \quad \tilde{h}_{13} \lessgtr 0 \text { for } \xi_{2} \lessgtr 0,
$$

which must be verified for any adopted $\tilde{f}(\xi)$ over the complete range of ordered $b_{r}(r=1,2,3)$.

\section{UNIAXIAL AND SHEAR RESPONSES}

In uniaxial compression at constant temperature $T$ along the $O x_{3}$ axis there are equal lateral stretches $\lambda_{1}=\lambda_{2}>1$, and the axial stretch (a compression) is $\lambda_{3}=\lambda_{1}^{-2}<1$. The model viscous law (Equation (2.1)) gives the non-zero diagonal relations

$$
\begin{aligned}
u\left(\xi_{1}\right) & =\frac{\hat{\sigma}_{11}}{2 \mu \tilde{D}_{11}}=\frac{\hat{\sigma}_{22}}{2 \mu \tilde{D}_{22}}=\frac{\hat{\sigma}_{33}}{2 \mu \tilde{D}_{33}} \\
& =\left[1+\frac{2}{3} \tilde{f}\left(\xi_{1}\right)+\frac{4}{3} \tilde{f}\left(\xi_{3}\right)+\frac{2}{3}\left(b_{1}+2 b_{1}^{-2}\right) \tilde{g}(\eta)\right]^{-1},
\end{aligned}
$$

where $u(0)=1$ and

$$
\begin{aligned}
\xi_{1} & =b_{1}-b_{1}^{-1} \geq 0, \quad \xi_{3}=b_{1}^{-2}-b_{1}^{2} \leq 0, \\
\eta & =\sqrt{\left(2 b_{1}+b_{1}^{-2}-1\right)^{2}-4} \geq 0
\end{aligned}
$$

As $b_{1} \rightarrow \infty$, then $\xi_{1} \rightarrow \infty, \xi_{3} \rightarrow-\infty$ and $\eta \sim 2 b_{1} \rightarrow \infty$, and $u\left(\xi_{1}\right) \rightarrow A ; A^{-1}$ is the enhancement factor in uniaxial compression. Eliminating $g(\eta)$ by Equation(2.6),

$$
2 \tilde{f}^{\mathrm{e}}(\infty)-\frac{4}{3} \tilde{f}^{\mathrm{o}}(\infty)=A^{-1}-1
$$

In a simple shear strain $\kappa \geq 0$ at constant temperature and constant strain rate $D_{13}=\frac{1}{2} \dot{\gamma}=\frac{1}{2} a(T) \dot{\tilde{\gamma}}>0$, other $D_{i j}=0$, the strain tensor $\mathbf{B}$ has the non-zero components $B_{11}=1+\kappa^{2}, B_{22}=B_{33}=1$ and $B_{13}=B_{31}=\kappa$, and the deviatoric-stress tensor has axial components $\hat{\sigma}_{11}, \hat{\sigma}_{22}, \hat{\sigma}_{33}$, where $\hat{\sigma}_{11}+\hat{\sigma}_{22}+\hat{\sigma}_{33}=0$, in addition to the direct shear components $\hat{\sigma}_{13}=\hat{\sigma}_{31}$. The principal values of $\mathbf{B}$, $b_{r}(r=1,2,3)$, the associated principal vectors $\mathbf{e}^{(r)}$, and the invariants $\xi_{r}, \eta=\eta_{s}$, are given by

$$
b_{2}=1,2 b_{1}=2 b_{3}^{-1}=2+\kappa^{2}+\kappa \sqrt{\kappa^{2}+4},
$$

$$
\mathbf{e}^{(2)}=(0,1,0), \quad e_{1}^{(1)}=e_{3}^{(3)}=\left[1+\left(\frac{\kappa}{b_{1}-1}\right)^{2}\right]^{-\frac{1}{2}}
$$

$$
\begin{gathered}
e_{3}^{(1)}=-e_{1}^{(3)}=\left[1+\left(\frac{b_{1}-1}{\kappa}\right)^{2}\right]^{-\frac{1}{2}}, \\
\xi_{2}=0, \quad \xi_{1}=-\xi_{3}=\eta_{s}=\kappa \sqrt{\kappa^{2}+4} .
\end{gathered}
$$

The model law (Equation (2.1)) then has the three 
independent relations

$$
\begin{aligned}
2 \mu \tilde{D}_{11}= & \hat{\sigma}_{11}+\tilde{f}\left(\eta_{s}\right) A_{11}^{(1)}+\tilde{f}(0) A_{11}^{(2)}+\tilde{f}\left(-\eta_{s}\right) A_{11}^{(3)} \\
& \quad-2 C_{11} \tilde{f}^{\mathrm{o}}\left(\eta_{s}\right) / \eta_{s}, \\
2 \mu \tilde{D}_{33}= & \hat{\sigma}_{33}+\tilde{f}\left(\eta_{s}\right) A_{33}^{(1)}+\tilde{f}(0) A_{33}^{(2)}+\tilde{f}\left(-\eta_{s}\right) A_{33}^{(3)} \\
& \quad-2 C_{33} \tilde{f}^{\mathrm{o}}\left(\eta_{s}\right) / \eta_{s}, \\
\mu \dot{\tilde{\gamma}}= & \hat{\sigma}_{13} / s(\kappa) \\
= & \hat{\sigma}_{13}+\tilde{f}\left(\eta_{s}\right) A_{13}^{(1)}+\tilde{f}\left(-\eta_{s}\right) A_{13}^{(3)}-2 C_{13} \tilde{f}^{\mathrm{o}}\left(\eta_{s}\right) / \eta_{s},
\end{aligned}
$$

where

$$
\begin{gathered}
C_{11}=\left[4\left(1+\kappa^{2}\right) \hat{\sigma}_{11} / 3\right]-\left(2 \hat{\sigma}_{33} / 3\right) \\
+\left(2 \kappa \hat{\sigma}_{13} / 3\right)-\left(2 \hat{\sigma}_{22} / 3\right), \\
C_{22}=\left[-2\left(1+\kappa^{2}\right) \hat{\sigma}_{11} / 3\right]-\left(2 \hat{\sigma}_{33} / 3\right) \\
\quad-\left(4 \kappa \hat{\sigma}_{13} / 3\right)+\left(4 \hat{\sigma}_{22} / 3\right), \\
C_{33}=\left[-2\left(1+\kappa^{2}\right) \hat{\sigma}_{11} / 3\right]+\left(4 \hat{\sigma}_{33} / 3\right) \\
+\left(2 \kappa \hat{\sigma}_{13} / 3\right)-\left(2 \hat{\sigma}_{22} / 3\right), \\
C_{13}=C_{31}=\kappa\left(\hat{\sigma}_{11}+\hat{\sigma}_{33}\right)+\left(2+\kappa^{2}\right) \hat{\sigma}_{13}, \\
A_{11}^{(2)}=A_{33}^{(2)}=-2 \hat{\sigma}_{22} / 3, \quad A_{22}^{(2)}=4 \hat{\sigma}_{22} / 3,
\end{gathered}
$$

and for $(s=1,3)$ :

$$
\begin{aligned}
A_{11}^{(s)}= & \left(4 e_{1}^{(s)} e_{1}^{(s)} \hat{\sigma}_{11} / 3\right)-\left(2 e_{3}^{(s)} e_{3}^{(s)} \hat{\sigma}_{33} / 3\right) \\
& +\left(2 e_{1}^{(s)} e_{3}^{(s)} \hat{\sigma}_{13} / 3\right), \\
A_{22}^{(s)}= & \left(-2 e_{1}^{(s)} e_{1}^{(s)} \hat{\sigma}_{11} / 3\right)-\left(2 e_{3}^{(s)} e_{3}^{(s)} \hat{\sigma}_{33} / 3\right) \\
& -\left(4 e_{1}^{(s)} e_{3}^{(s)} \hat{\sigma}_{13} / 3\right), \\
A_{33}^{(s)}= & \left(-2 e_{1}^{(s)} e_{1}^{(s)} \hat{\sigma}_{11} / 3\right)+\left(4 e_{3}^{(s)} e_{3}^{(s)} \hat{\sigma}_{33} / 3\right) \\
& +\left(2 e_{1}^{(s)} e_{3}^{(s)} \hat{\sigma}_{13} / 3\right), \\
A_{13}^{(s)}= & A_{31}^{(s)}=e_{1}^{(s)} e_{3}^{(s)}\left(\hat{\sigma}_{11}+\hat{\sigma}_{33}\right)+\hat{\sigma}_{13} .
\end{aligned}
$$

Setting $\tilde{D}_{11}=\tilde{D}_{33}=0$ provides two linear relations to eliminate $\hat{\sigma}_{11}$ and $\hat{\sigma}_{33}$, and in turn $\hat{\sigma}_{22}$, in terms of $\hat{\sigma}_{13}$. Then Equation (3.9) can be expressed as a linear relation between $\dot{\tilde{\gamma}}$ and $\hat{\sigma}_{13}$, but involving $\tilde{f}\left(\eta_{s}\right)$ and $\tilde{f}\left(-\eta_{s}\right)$ in a complicated manner; the derivation was not pursued by Morland and Staroszczyk (2003).

We now show that this coupled system does lead to a simple explicit relation between $\dot{\tilde{\gamma}}$ and $\hat{\sigma}_{13}$, which can then be combined with the uniaxial relation (3.1) to relate the fabric function to the uniaxial and shear responses, as done for the stress formulation by Morland and Staroszczyk (2003). Ignoring the systematic procedure described above, we first determine the coefficients of $\hat{\sigma}_{13}$ in Equations (3.7) and (3.8) for $\tilde{D}_{11}$ and $\tilde{D}_{33}$. Collecting the relevant terms from Equations (3.10-3.18), it is found that both coefficients are

$$
\frac{2}{3}\left[\tilde{f}\left(\eta_{s}\right) e_{1}^{(1)} e_{3}^{(1)}+\tilde{f}\left(-\eta_{s}\right) e_{1}^{(3)} e_{3}^{(3)}-\frac{2 \kappa}{\eta_{s}} \tilde{f}^{\mathrm{o}}\left(\eta_{s}\right)\right] .
$$

Now, from Equations (3.4-3.6), it follows that

$$
e_{1}^{(1)} e_{3}^{(1)}=-e_{1}^{(3)} e_{3}^{(3)}=\kappa / \eta_{s},
$$

so that the coefficient (3.19) is a multiple of $\tilde{f}^{\circ}\left(\eta_{s}\right)$ and is identically zero. Thus, setting $\tilde{D}_{11}=\tilde{D}_{33}=0$ yields two non-singular, homogeneous, linear equations for $\hat{\sigma}_{11}$ and $\hat{\sigma}_{33}$, which implies that $\hat{\sigma}_{11}=\hat{\sigma}_{33}=0$, and hence Equation (3.9) reduces to

$$
s(\kappa)=\left[1+2 \tilde{f}^{\mathrm{e}}\left(\eta_{s}\right)-2\left(\kappa^{2}+2\right) \tilde{f}^{\mathrm{o}}\left(\eta_{s}\right) / \eta_{s}\right]^{-1} .
$$

As $\kappa \rightarrow \infty$, then $\eta_{s} \sim \kappa^{2} \rightarrow \infty$ and $s(\kappa) \rightarrow S$, where $S^{-1}$ is the enhancement factor in simple shear, and the shear relation (3.21) has the limit

$$
2\left[\tilde{f}^{\mathrm{e}}(\infty)-\tilde{f}^{\mathrm{o}}(\infty)\right]=S^{-1}-1
$$

Combining Equation (3.22) with the axial limit relation (3.3) then determines the fabric response function limit values

$$
\begin{aligned}
& \tilde{f}^{\mathrm{e}}(\infty)=\left(3 A^{-1}-2 S^{-1}-1\right) / 2, \\
& \tilde{f}^{\mathrm{o}}(\infty)=-3\left(S^{-1}-A^{-1}\right) / 2 .
\end{aligned}
$$

\section{GORRELATIONS}

We adopt the Morland and Staroszczyk (2003) idealized monotonic responses for the uniaxial and shear responses which prescribe $u\left(\xi_{1}\right)$ and $s(\kappa)$, respectively, by

$$
\begin{aligned}
& u_{\mathrm{p}}\left(b_{1}\right)=1+(A-1)\left\{1-\exp \left[-\left(b_{1}-1\right) / b_{*}\right]^{2}\right\}, \\
& s_{\mathrm{p}}\left(\kappa^{2}\right)=1+(S-1)\left[1-\exp \left(-\kappa^{2} / k_{*}\right)\right],
\end{aligned}
$$

where $b_{*}$ and $k_{*}$ are the scales of $b_{1}$ and $\kappa^{2}$, respectively, over which $u_{\mathrm{p}}\left(b_{1}\right)$ and $s_{\mathrm{p}}\left(\kappa^{2}\right)$ make a significant change. These prescriptions satisfy the limits at $b_{1}=1$ and $\kappa=0$, and as $b_{1}$ and $\kappa \rightarrow \infty$, and have zero and non-zero derivatives, respectively, with respect to their arguments at $b_{1}=1$ and $\kappa^{2}=0$ as required by Equations (3.1) and (3.21). Now $s(\kappa)$ given by Equation (3.21) can be matched exactly with the prescribed $s_{\mathrm{p}}\left(\kappa^{2}\right)$ by setting

$$
\tilde{f}^{\mathrm{o}}\left(\eta_{s}\right)=\frac{\eta_{s}}{\sqrt{\eta_{s}^{2}+4}}\left[\tilde{f}^{\mathrm{e}}\left(\eta_{s}\right)+0.5-0.5 \tilde{s}_{\mathrm{p}}\left(\eta_{s}^{2}\right)\right]
$$

for $\eta_{s} \geq 0$, which in turn expresses $\tilde{f}(\xi)$ in terms of $\tilde{f}^{\mathrm{e}}(\xi)$ and $\tilde{s}_{\mathrm{p}}\left(\xi^{2}\right)$ for all $\xi$, where

$$
\tilde{s}_{\mathrm{p}}\left(\eta_{s}^{2}\right)=s_{\mathrm{p}}\left(\kappa^{2}\right), \quad \kappa^{2}=\sqrt{\eta_{s}^{2}+4}-2 .
$$

The required derivative condition $(2.8), \tilde{f}^{o^{\prime}}(0)=0.5 \tilde{f}(0)$, is automatically satisfied by Equation (4.2). While there is still a controversy whether the limit value $A$ is greater or less than unity, we consider both the "warm-" and "cold-ice" parameters adopted by Morland and Staroszczyk (2003), based respectively on laboratory tests by Budd and Jacka (1989), and modifications of estimates by Mangeney and others (1996) from Greenland Icecore Project (GRIP) and Greenland Ice Sheet Project 2 (GISP2) ice-core data:

warm ice: $A=1 / 3, S=1 / 8, \quad b_{\star}=k_{\star}=0.5$,

$$
\tilde{f}^{\mathrm{o}}(\infty)=-7.5, \quad \tilde{f}^{\mathrm{e}}(\infty)=-4, \quad \tilde{f}(\infty)=-11.5,
$$

cold ice: $A=3, \quad S=1 / 5, \quad b_{\star}=k_{\star}=4$,

$$
\tilde{f}^{\circ}(\infty)=-7, \quad \tilde{f}^{\mathrm{e}}(\infty)=-5, \quad \tilde{f}(\infty)=-12 .
$$

The fabric function limits are given by Equations (3.23) and (2.7).

Correlation of the predicted uniaxial and shear responses $u\left(\xi_{1}\right)$ and $s(\kappa)$ with the prescribed responses $u_{\mathrm{p}}\left(b_{1}\right)$ and $s_{\mathrm{p}}\left(\kappa^{2}\right)$, respectively, determines the corresponding fabric response function $\tilde{f}(\xi)$ in the assumed model. We apply the weighted least-squares difference minimization used by Morland and Staroszczyk (2003) in the stress formulation. As there, the best correlation for "warm ice" was obtained by the combined uniaxial and shear response minimization, and for "cold ice" by the uniaxial minimization with $s(\kappa)$ matched exactly, adopting the same fabric response function representations. Since there is no apparent critical value of $\xi(b)$ during the fabric evolution, it is expected that the func- 


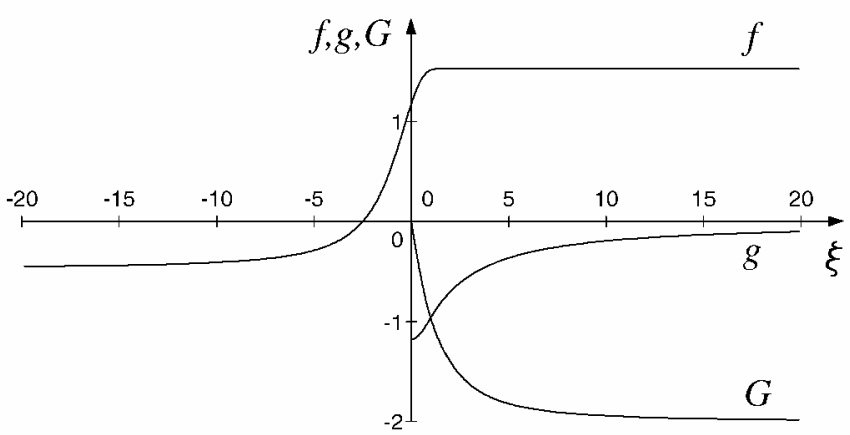

Fig. 1. Fabric functions $f(\xi), g(\xi)$ and $G(\xi)$ for warm ice.

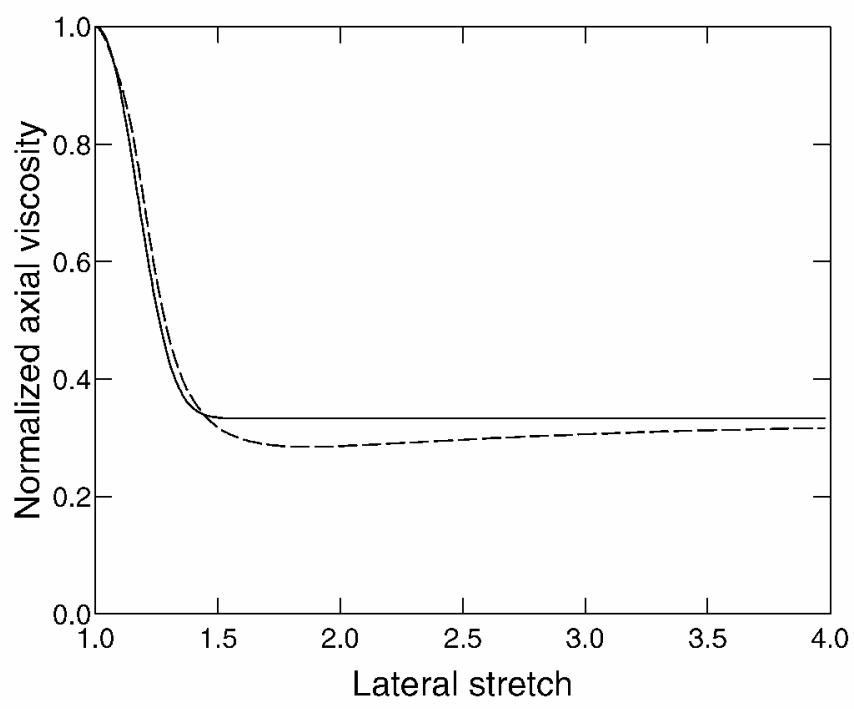

Fig. 2. Calculated normalized axial viscosity $u\left(\xi_{1}\right)$ (Equation (3.1)) vs lateral stretch $\lambda_{1}$ (dashed line) compared to prescribed response ( solid line) for warm ice.

tion $\tilde{f}(\xi)$ should be monotonic, satisfying the limit values given by Equations (4.4) and (4.5). Monotonicity of $\tilde{f}(\xi)$ ensures monotonicity of $\tilde{f}^{\circ}(\xi)$ and $\tilde{G}(\eta)$, but not of $\tilde{g}(\eta)$. For the combined correlation, the representation is

$$
\begin{aligned}
\tilde{f}(\xi) & =\tilde{f}(\infty)-2 \tilde{f}^{\circ}(\infty) \exp [-\alpha q(b)], \\
q(b) & =\sum_{n=1}^{N} a_{n} b^{c_{n}} \\
2 b & =\xi+\sqrt{\xi^{2}+4}, \quad \alpha, a_{n}, c_{n}>0,
\end{aligned}
$$

which is necessarily monotonic. The derivative condition (2.8) at $\xi=0, b=1$ becomes

$$
2 \tilde{f}^{\circ}(\infty)\left[1+\alpha q^{\prime}(1)\right] \exp [-\alpha q(1)]=\tilde{f}(\infty),
$$

which has a unique positive root $\alpha$, leaving $2 N$ free parameters $a_{n}$ and $c_{n}$ in the representation (4.6). For the uniaxial correlation, with the shear response matched identically, the representation for the even part $\tilde{f}^{\mathrm{e}}(\xi)$ is

$$
\tilde{f}^{\mathrm{e}}(\xi)=\tilde{f}^{\mathrm{e}}(\infty)+\tilde{f}^{\mathrm{o}}(\infty) \sum_{n=1}^{N} \frac{a_{n}}{1+c_{n}\left(2 \xi / b_{*}\right)^{2 n}},
$$

with $c_{n}>0$, but allowing some of the coefficients $a_{n}$ to be negative, so monotonicity of $\tilde{f}^{\mathrm{e}}(\xi)$ in $\xi \geq 0$ is not ensured, nor, in general, is that of $\tilde{f}(\xi)$. The representation (4.8) also has two $N$-free parameters $a_{n}$ and $c_{n}$.

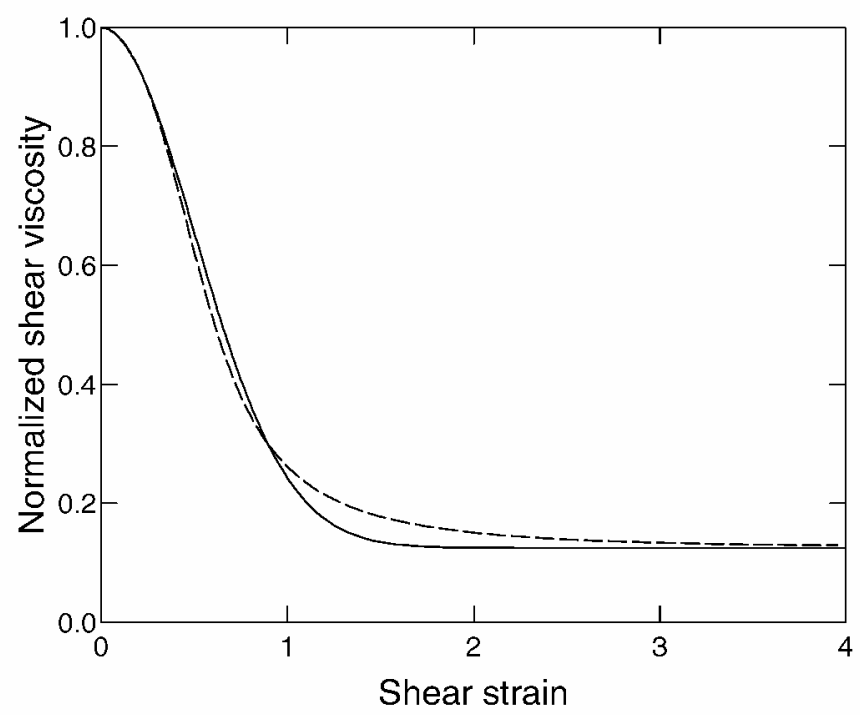

Fig. 3. Calculated normalized shear viscosity $s(\kappa)$ (Equation (3.21)) vs shear strain $\kappa$ (dashed line) compared to prescribed response (solid line) for warm ice.

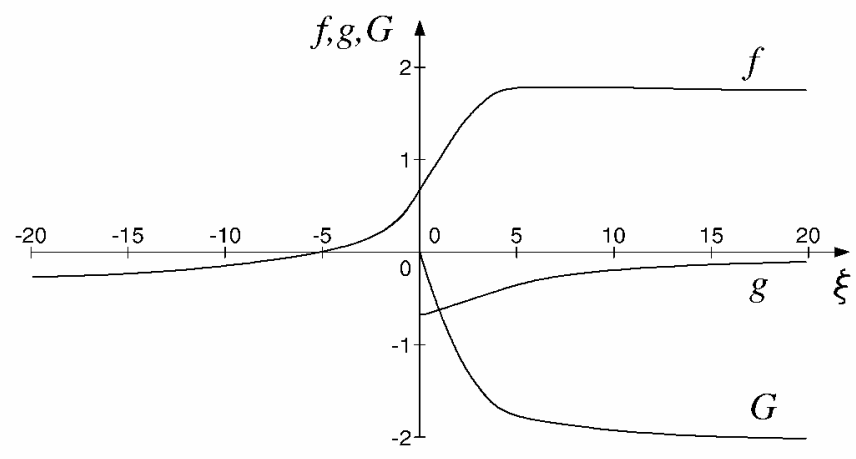

Fig. 4. Fabric functions $f(\xi), g(\xi)$ and $G(\xi)$ for cold ice.

The best correlations obtained with moderate $N$ are as follows. For "warm ice", the coefficients in Equation (4.6) are

$$
\begin{aligned}
\alpha & =1, \quad a_{1}=1.6467, \quad a_{2}=0.0787, \\
c_{1} & =1.7440, \quad c_{2}=5.4995 .
\end{aligned}
$$

Figure 1 shows the corresponding fabric response functions, all monotonic, and Figures 2 and 3 show the prescribed and predicted uniaxial and shear responses, respectively. The maximum uniaxial and shear differences are, respectively, 0.057 compared to $A=0.333$, and 0.045 compared to $S=$ 0.2. For "cold ice", the coefficients in Equation (4.8) are

$$
\begin{aligned}
& a_{1}=0.2263, \quad a_{2}=-0.2331, \quad a_{3}=0.0356, \\
& a_{4}=-0.0975, \quad a_{5}=0.0296 ; \\
& c_{1}=0.0827, \quad c_{2}=1.5143, \quad c_{3}=0.000014, \\
& c_{4}=0.0167, \quad c_{5}=14.5080 .
\end{aligned}
$$

Figure 4 shows the corresponding fabric response functions, all monotonic, Figure 5 shows the prescribed and predicted uniaxial response, and Figure 6 the identically matched prescribed shear response. The maximum uniaxial difference is 0.15 compared to $A=3$. In both cases, the validity inequalities (2.10) were verified numerically over the wide range

$$
1001 \geq b_{1} \geq 1, \quad 0.01 \leq b_{2} \leq b_{1} \quad\left(b_{3} \leq b_{2}\right) .
$$




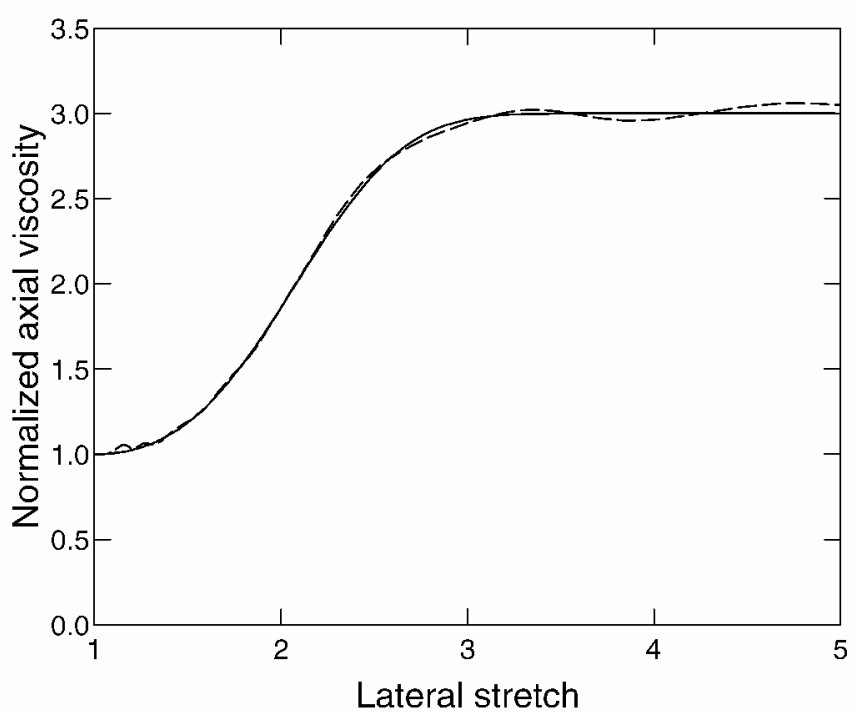

Fig. 5. Calculated normalized axial viscosity $u\left(\xi_{1}\right)$ (Equation (3.1)) vs lateral stretch $\lambda_{1}$ (dashed line) compared to prescribed response (solid line) for cold ice.

\section{CONCLUSIONS}

We have presented a strain-rate formulation of an orthotropic viscous law for evolving fabric in polar ice, and shown that explicit relations between the primary strain rate and stress can be obtained for both uniaxial compression and simple shear responses. These allow correlation with idealized uniaxial compression and simple shear responses, for both "warm-" and "cold-ice" properties, to determine the fabric response coefficient functions which yield good approximations to those prescribed responses. We therefore now have models for both stress and strain-rate formulations which determine, to a close approximation, the same uniaxial compression and simple shear responses.

\section{AGKNOWLEDGEMENT}

This research was supported by a U.K. Engineering and Physical Sciences Research Council grant, "Evolving Anisotropy of Ice Sheet Flows".

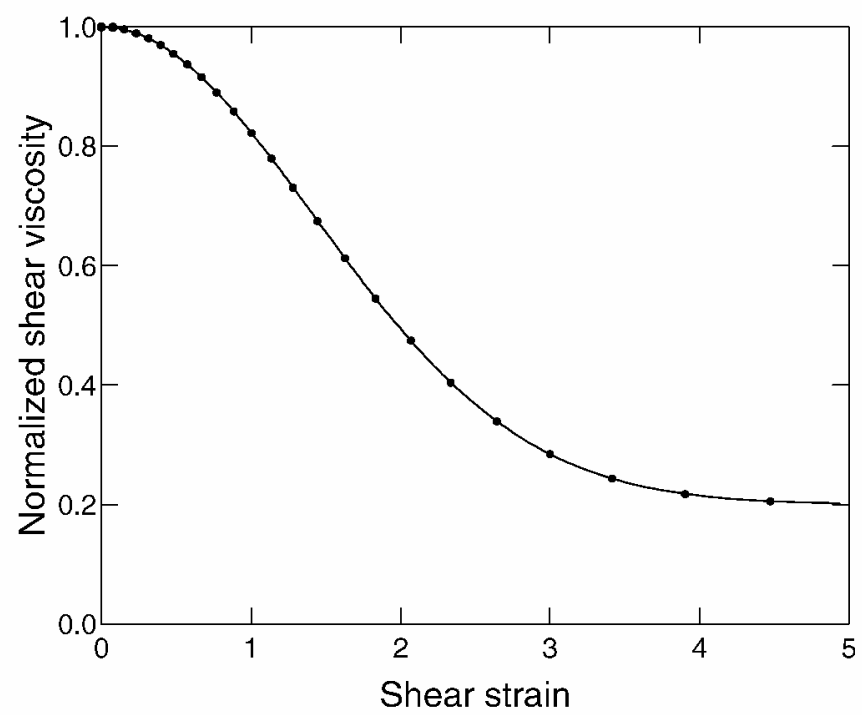

Fig. 6. Calculated normalized shear viscosity $s(\kappa)$ (Equation (3.21)) vs shear strain $\kappa$ (dots) identical to prescribed response (solid line) for cold ice.

\section{REFERENGES}

Azuma, N. and K. Goto-Azuma. 1996. An anisotropic flow law for ice-sheet ice and its implications. Ann. Glaciol., 23, 202-208.

Budd, W. F. and T. H. Jacka. 1989. A review of ice rheology for ice sheet modelling. Cold Reg. Sci. Technol., 16(2), 107-144.

Castelnau, O., P. Duval, R. Lebensohn and G. R. Canova. 1996. Viscoplastic modeling of texture development in polycrystalline ice with a self-consistent approach: comparison with bound estimates. F. Geophys. Res., 101(B6), $13,851-13,868$.

Gagliardini, O. and J. Meyssonnier. 1999. Analytical derivations for the behaviour and fabric evolution of a linear orthotropic ice polycrystal. 7. Geophys. Res., 104(B8), 17,797-17,809.

Gödert, G. and K. Hutter. 1998. Induced anisotropy in large ice shields: theory and its homogenization Continuum Mech. Thermodyn., 10(5), 293-318.

Mangeney, A., F. Califano and O. Castelnau. 1996. Isothermal flow of an anisotropic ice sheet in the vicinity of an ice divide. 7. Geophys. Res., 101(B12), 28,189-28,204.

Meyssonnier, J. and A. Philip. 1996. A model for the tangent viscous behaviour of anisotropic polar ice. Ann. Glaciol., 23, 253-261.

Morland, L. and R. Staroszczyk. 1998. Viscous response of polar ice with evolving fabric. Continuum Mech. Thermodyn., 10(3), 135-152.

Morland, L.W. and R. Staroszczyk. 2003. Stress and strain-rate formulations for fabric evolution in polar ice. Continuum Mech. Thermodyn., 15(1), $55-71$

Staroszczyk, R. and L.W. Morland. 2000. Orthotropic viscous response of polar ice. 7. Eng. Math., 37(1-3), 191-209.

Staroszczyk, R. and L.W. Morland. 2001. Strengthening and weakening of induced anisotropy in polar ice. Proc. R. Soc. London, Ser. A, 457(2014), 2419-2440.

Svendsen, B. and K. Hutter. 1996. A continuum approach for modelling induced anisotropy in glaciers and ice sheets. Ann. Glaciol., 23, 262-269.

Van der Veen, C. J. and I. M. Whillans. 1994. Development of fabric in ice. Cold Reg. Sci. Technol., 22(2), 171-195. 